\begin{tabular}{ccc}
\hline & International Journal of Engineering \& Technology, $7(3.29)(2018) 263-268$ \\
SPC & Website $: \frac{w w . s c i e n c e p u b c o . c o m / i n d e x . p h p / I J E T}{2}$ \\
Research paper & Technology \\
\hline
\end{tabular}

\title{
A review report on challenges and opportunities of edge, fog and cloud computing by employing IoT technology
}

\author{
Sk. Wasim Akram ${ }^{1}$ *, Dr. P. Rajesh ${ }^{2}$, Sk. Shama ${ }^{3}$ \\ ${ }^{I}$ Asst. Professor, Dept. of CSE, VVIT College, Guntur, India \\ ${ }^{2}$ Professor, Dept. of CSE, KLEF, Deemed to be University, Vaddeswaram, Guntur, India \\ ${ }^{3}$ Asst. Professor, Dept. of CSE, SRK Institute of technology, Enkipadu, Vijayawada, India \\ *Corresponding author E-mail: shaikwasimakram585@gmail.com
}

\begin{abstract}
In the future, various information and things will be connected to the network. People can now live more convenient and comfortable life where the things and information coordinated together. A world where things are connected to network is referred as IOT (Internet of Things). A huge amount of incomplete data is generated by IOT need to process and responded to very short time. This pose challenge of dealing with big data from many geometrically distributed data sources which are to be managed and processed. To achieve this objective, cloud computing is a treated as one of the popular choice due to its scalability, storage, computational and other capabilities. However current cloud models are not intended to handle the essentials of IOT- volume, variety, and velocity of data. Moreover, as the physical distance between cloud and user increases, transmission latency increases with it, increasing response time and stressing of the user. In addition to that, the processing speed in this environment is largely dependent on the performance of user device. The viable solution to these problems is identified as Edge Computing. The Edge Computing platform works by allowing some application processing to be performed by a small edge server position between the cloud and user, and crucially in a location physically closed to the user. This paper comprehensively presents various research trends that are available in Edge, Fog computing along with a comparison is made among Cloud. Particularly the architecture, characteristics, key technologies, potential applications, security issues and challenges of Edge, Fog and Cloud Computing are discussed and summarized.
\end{abstract}

Keywords: IOT; Cloud Computing; Fog Computing; Edge Computing; Software Defined Networking (SDN); Network Function Virtualization (NFV); Content Distributed Networking (CDN).

\section{Introduction}

According to National Institute of Science and Technology (NIST), the term 'Cloud computing' refers to computing services available to anyone online. It is a model for allowing convenient, on -demand access from anywhere, to a shared pool of computing resources. These can include server's storage, networking applications and services that can be rapidly and easily provisioned and released. In today's network world, there are numerous applications of cloud co. Many social websites like www.amazon.com, hotmail.com, facebook.com, linkedln.com etc. and search engines like google are utilizing the concept of cloud computing that gained huge popularity from research area to real time IoT applications by provides numerous services [1] to end users like: Software as a service (Saas), Platform as a service (Paas), Resource Pooling, On-demand selfservice, rapid elasticity and so on. A significant drawback of cloud computing is high latency due to large physical distance between cloud and user devices. Some other limitations and challenges [2] of cloud are: limited bandwidth, lack of protection mechanisms, identifying data location is difficult, data segregation is not possible and finally recovery and backup of data is also quite slow.

\subsection{Fog computing}

As cloud is suffered from various key issues like latency, mobility, security and privacy, which has motivated researchers to propose a new computing paradigm called Fog computing. Fog Computing [3] also known as Fogging or Cloudlets is a distributed computing infrastructure in which some application services are handled at the intermediate node of the core network called "Fog Nodes". A Fog node is physical device where the Fog computing is deployed for example: router, set-up box, cellular access points, smart meters etc. According to Cisco, due to its wide geographical distribution, the Fog computing is well suited for near real-time analytics [4] like Smart grid, smart city and smart vehicle etc. Fog computing involves a dense geographical distribution of network and provides a feature of location access. With this any unauthorized activity in the cloud network can be detected.

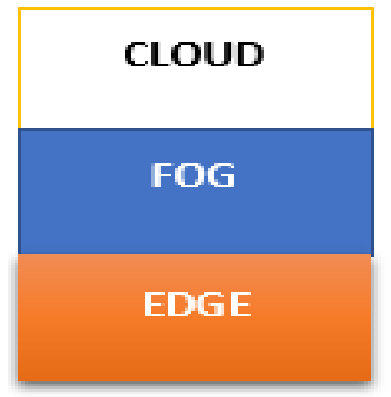

Fig. 1: Fog Acts as Intermediate Layer between Cloud and Edge. 
Fog Computing focuses and provide solution to several issues that includes, reduces network latency, low network traffic, connect to any kind of IoT device, saves lots of bandwidth, provide security to IoT devices, allow to perform analysis using Fog nodes [5] like setup box, LAN, router etc. It applies some business rules to decide what data to process locally and what to send to cloud. Some mechanism like pattern recognition, anomaly detection and prediction are used to process huge amount of data to get some hidden patterns which are further helpful in making decisions. When to consider Fog Computing is, where there exists Thousands and millions of things across a large geographic area are generating data, it is necessary to analyze and act on the data in less than a second.

\subsection{Edge computing}

A new buzz word in computing technology is Edge Computing [6], which is a subsection of Fog Computing. In Edge computing, instead of sending all its data over the internet to the on-premise data center or cloud, the data is delivered to nearby computing devices such as a computer, smart phones, laptops or a gate way networking device for analysis. A device which processing the data itself is treated as a mini data center. Edge computing is one of widespread approach by pushing intelligence at data generating devices itself i.e. sensors, controllers, and other connected devices gather and analyze IoT data themselves and put them into action. It processes many real time applications like video streaming, Gaming, Health care etc.

The major benefits of Edge computing are minimized Latency down to milliseconds, Lower Data management costs, Reduce load on internet networks and improved privacy and security. Edge Computing facilitates analytics and knowledge generation to occur at the source of the data instead of holding the processing power in cloud is known as "Edge analytics". It is an approach where the data collection and analysis are performed at sensor, network switch, or other device instead of waiting for data to be sent back to a centralized data store. The below diagram (Fig 2) tells that current Edge Computing is a subset of Fog Computing domain [7].

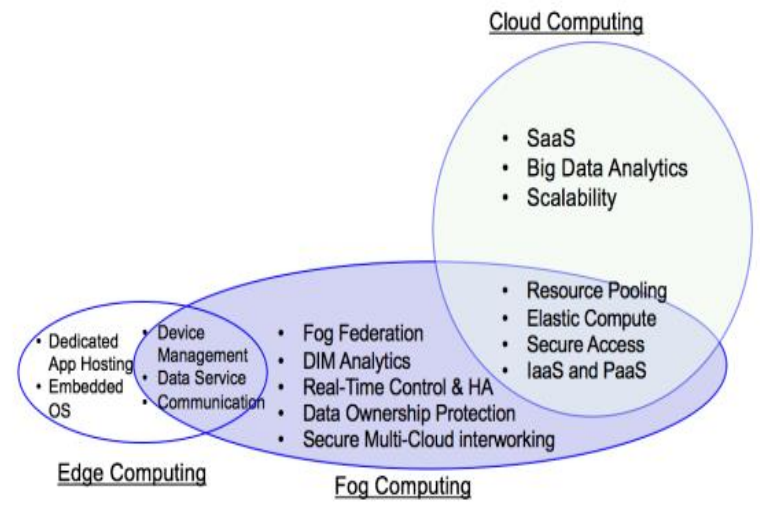

Fig. 2: Similarities and Dissimilarities of Cloud, Fog and Edge Computing

Fog computing and Edge computing both are similar concepts, but some dissimilarity is there: Edge computing brings compute, storage and networking resources directly to the end points i.e. the data generated devices itself have processing power and communication capabilities (Intelligence) with constrained resources. The Edge computing platform allows providing rapid response while processing the real-time application at near locations of user devices. Whereas the Fog computing brings compute, storage and networking resources are close to the end points as possible. Fog is away from cloud but does not include the end points. In Fog Computing the data processing power (Intelligence) is done at Fog nodes like Local area networks, IoT gateway and other devices where the data get processed. The below figure explains the architecture of Cloud, Fog and Edge Computing Paradigm:

\section{Key characteristics of cloud, fog and edge computing}

With quick development of IoT Technology and its applications, the existed cloud computing architecture is encountering several challenges. Some of the potential Key characteristics that differ Edge computing, Fog Computing and Cloud Computing are listed and shown in TABLE1.

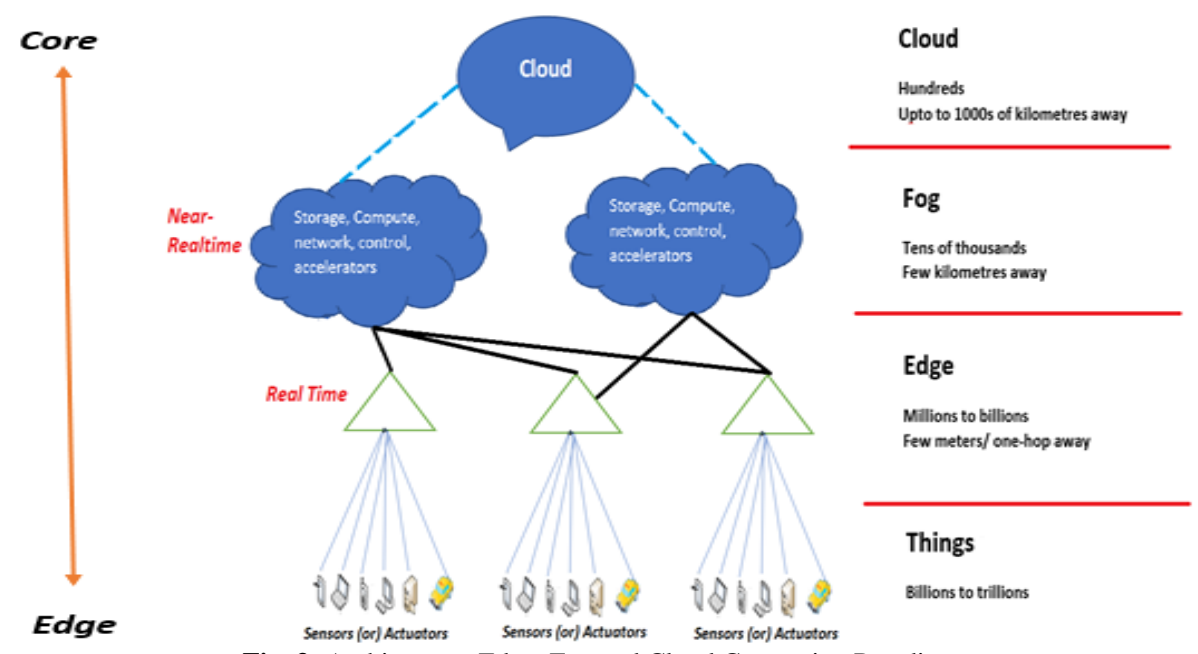

Fig. 3: Architecture: Edge, Fog and Cloud Computing Paradigm.

Table 1: Comparison of Characteristics among Cloud, Fog and Edge Computing

\begin{tabular}{lllll}
\hline S. No & Key Features & Cloud Computing & Fog Computing & Edge Computing \\
\hline 1 & $\begin{array}{l}\text { Computation of data } \\
\text { and services }\end{array}$ & $\begin{array}{l}\text { Data and applications are pro- } \\
\text { cessed in a cloud, which is time } \\
\text { consuming for large data. }\end{array}$ & $\begin{array}{l}\text { Reduction in data movement } \\
\text { across the network resulting in } \\
\text { reduced congestion }\end{array}$ & $\begin{array}{l}\text { Here data Processing is done at } \\
\text { data generating devices them- } \\
\text { selves which reduces network } \\
\text { congestion }\end{array}$ \\
$\begin{array}{l}\text { Architecture and Geo- } \\
\text { distribution }\end{array}$ & $\begin{array}{l}\text { It is a Central processing-based } \\
\text { model architecture }\end{array}$ & $\begin{array}{l}\text { It is a Distributed Computing } \\
\text { model }\end{array}$ & $\begin{array}{l}\text { Eding, so it is also treated as Dis- } \\
\text { tributed model } \\
\text { Highly supported }\end{array}$ \\
\hline
\end{tabular}




\begin{tabular}{|c|c|c|c|c|}
\hline 4 & Response time & $\begin{array}{l}\text { Slow response time and scalabil- } \\
\text { ity problems because of depend- } \\
\text { ing servers that are located at re- } \\
\text { mote places }\end{array}$ & $\begin{array}{l}\text { Improves system response time } \\
\text { by eliminating a round trip to the } \\
\text { cloud for analysis }\end{array}$ & $\begin{array}{l}\text { Provides high response time } \\
\text { because the data service is } \\
\text { done at edge of network }\end{array}$ \\
\hline 5 & Scalability & Low & \multirow{2}{*}{$\begin{array}{l}\text { Higher than Cloud Computing } \\
\text { Storage is high when compared } \\
\text { to edge devices }\end{array}$} & Higher than Fog Computing \\
\hline 6 & Storage & Unlimited & & Limited \\
\hline 7 & Analytics & $\begin{array}{l}\text { Cloud can collect data from vari- } \\
\text { ous fog devices to perform pro- } \\
\text { cessing }\end{array}$ & $\begin{array}{l}\text { Fog node collects data from vari- } \\
\text { ous edge devices for analysis, } \\
\text { machine learning and anomaly } \\
\text { detection. }\end{array}$ & $\begin{array}{l}\text { Analytics scoped to a single } \\
\text { device }\end{array}$ \\
\hline 8 & Scope of the network & Complete network scope & $\begin{array}{l}\text { Complete network scope but it is } \\
\text { limited to domain }\end{array}$ & Limited network scope \\
\hline 9 & Usage of bandwidth & $\begin{array}{l}\text { It requires higher bandwidth, } \\
\text { which can increase costs }\end{array}$ & $\begin{array}{l}\text { It avoids the need for costly } \\
\text { bandwidth additions by offload- } \\
\text { ing gigabytes of network traffic } \\
\text { from the core network. }\end{array}$ & It consumes less bandwidth \\
\hline 10 & Security & $\begin{array}{l}\text { There is less security for data be- } \\
\text { cause the physical distance be- } \\
\text { tween Cloud and end user is more. }\end{array}$ & $\begin{array}{l}\text { Improved security of encrypted } \\
\text { of data as it stays closer to end } \\
\text { user }\end{array}$ & $\begin{array}{l}\text { Security is high, but it is lim- } \\
\text { ited to devices }\end{array}$ \\
\hline 11 & Location Awareness & $\begin{array}{l}\text { Location awareness is not possi- } \\
\text { ble }\end{array}$ & Location awareness is possible & Location awareness is possible \\
\hline 12 & Latency & High & Low & $\begin{array}{l}\text { Very low i.e. Data is analyzed } \\
\text { at the local level }\end{array}$ \\
\hline 13 & $\begin{array}{l}\text { Number of server } \\
\text { nodes }\end{array}$ & $\begin{array}{l}\text { Cloud Computing contains few } \\
\text { number of server nodes for pro- } \\
\text { cessing the data }\end{array}$ & $\begin{array}{l}\text { Number of server nodes are large } \\
\text { for processing the real time data }\end{array}$ & $\begin{array}{l}\text { Each device Process its own } \\
\text { data and acts as a mini data } \\
\text { center }\end{array}$ \\
\hline 14 & Jitter & High & Low & Very low \\
\hline
\end{tabular}

\section{Emerging trends in fog computing and edge computing}

The Cloud, Fog and Edge computing can be incorporated with the emerging Computation, networking and storage technologies with a layered architecture as shown in below figure:

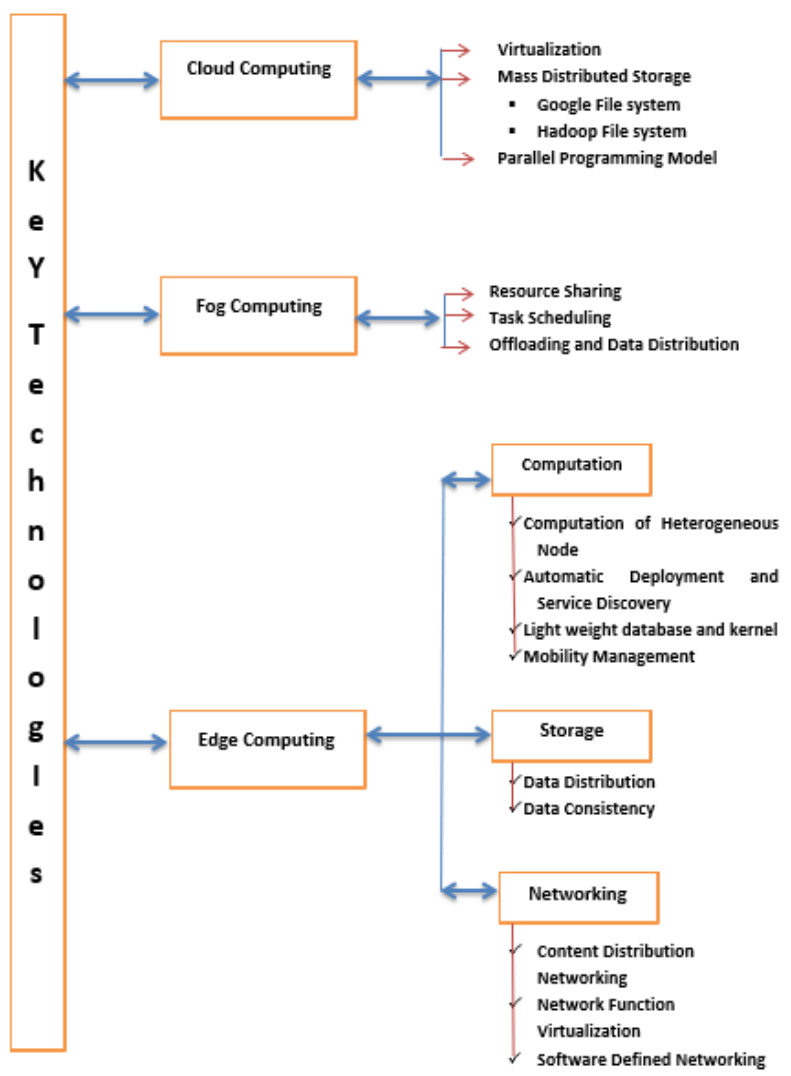

Fig. 4: Key Technologies of Edge, Fog and Cloud Computing.

\subsection{Technologies used in cloud computing}

This paradigm utilizes many technologies [8] like Virtualization, Mass storage management, parallel programming model that are responsible for Deploying the computing resources, adopts distributed storage to save the data redundantly using Hadoop Distributed File System (HDFS) and Google File System (GFS), To utilize the computing resources efficiently, it provides programming model to schedule the tasks and execute them parallel. Cloud uses a popular parallel programming model called 'Map-Reduce' which was developed by google to enable parallelism, data distribution and identify fault tolerance in the system.

\subsection{Technologies used in fog computing}

Fog Computing introduces many application specific algorithms [9] that focuses on Computation, data storage, data distribution and consumption of energy in a large scale distributed computing system. Fog Computing uses Resource sharing algorithm that enables to describe how the resources are shared among fog nodes in a fog domain to perform user's computational tasks. Fog utilizes task scheduling algorithms to decide which tasks to be executed in fog nodes and which task to be send to the cloud to make future analysis i.e.; it simply focuses on how to manage the tasks that are raised from end-user devices. One of the main key strategy of Fog system is, it minimizes the energy consumption or latency in the system by performing the computations offloading and balance the work load among fog nodes by using load Redistribution Mechanism.

\subsection{Technologies used in edge computing}

Edge Computing is an open platform where the core capabilities [10] like computation, networking, and storage are performed at edge of the network near the data generated sources. This can perform critical functionalities like application intelligence, real time services, data optimization and finally provide privacy and security to the user devices. To implement Edge Computing, some key aspects must be considered:

- Computation

- Storage

- Communication/ Networking

\subsubsection{Computation}

In Edge computing, most of the data is computed and stored away from the cloud i.e. Edge devices are responsible to perform computation nearer to end users without forwarding the data to cloud, that 
reduces network traffic and minimizes the latency. The Edge nodes are capable to meet technical challenges like Computation of Heterogenous nodes, Automatic Deployment, automatic troubleshooting, Intelligent Configuration and service discovery, one of the main challenge is that Edge Computing applications consumes less storage and computation resources (Light weight database and kernel) for processing the IoT data.

\subsubsection{Storage}

In Edge Computing, the data is stored at those devices where computation is performed. In the distributed environment, the data which is created by user devices are stored at local file storage systems of edge so that it reduces the computation delay and at the same it maintains data consistency to avoid undesired outcomes.

\subsubsection{Networking}

To simplify the network functions from traditional cloud to edge architecture, the networking world offers several popular approaches [11] [12]: Network Function Virtualization (NFV), Software Defined Networking (SDN), Content Distribution Networking $(\mathrm{CDN})$. Both SDN and NFV are used for network abstraction and make networking architectures more flexible and dynamic. However, they perform distinct roles in defining those architectures and the infrastructure they support.

\subsubsection{Network function virtualization (NFV)}

The basic idea of NFV is it separates network functions from firewalls, routers, load balancers, and other dedicated hardware devices that allows network services to be hosted on virtual machines. Every Virtual machine have a hypervisor, also termed a virtual machine manager, which allows multiple operating systems to share a single hardware processor. The hypervisor allows to control the network functions to perform services on a dedicated hardware where it contains standard $\mathrm{x} 86$ servers.

Fog Computing uses Hypervisor Virtualization for processing the application whereas Edge computing uses lightweight container technology for application delivery called docker. It is an open platform for developers and system admins to build ship and run distributed applications.

\subsubsection{Software defined networking (SDN)}

In Fog/Edge computing, as the local replacement of cloud (Fog nodes/ Edge devices) needs to synchronize frequently with cloud for data update and support. With a global network view, the cloud can manage the entire network using a SDN approach. SDN seeks to separate network control functions from network forwarding functions, SDN's networking control functions for routing, policy definition and applications run in a virtual machine somewhere on the network. SDN abstracts physical networking resources switches, routers and so on - and moves decision making to a virtual network control plane. In this approach, the control plane decides where to send traffic, while the hardware continues to direct and handle the traffic.

\subsubsection{Content distribution networking (CDN)}

A Content Distribution network (or) Content Delivery Network is a geographically distributed network of proxy servers and their data centers. A CDN is especially used in Edge Computing, to distribute services spatially relative to end users that provide high performance and high availability. $\mathrm{CDN}$ provides diverse types of content delivery services through internet that includes applications like Ecommerce, portals, social networks, live streaming media, web objects, downloadable objects, etc.

\section{Applications and challenges of fog and edge computing}

The Fog and Edge computing has certain characteristics that focuses on mobility of devices, Heterogeneity, location awareness of devices and so on. A list of Challenges, Characteristics [13], Benefits, Applications [14] and disadvantages of each computing model is shown in Fig5, Fig6.

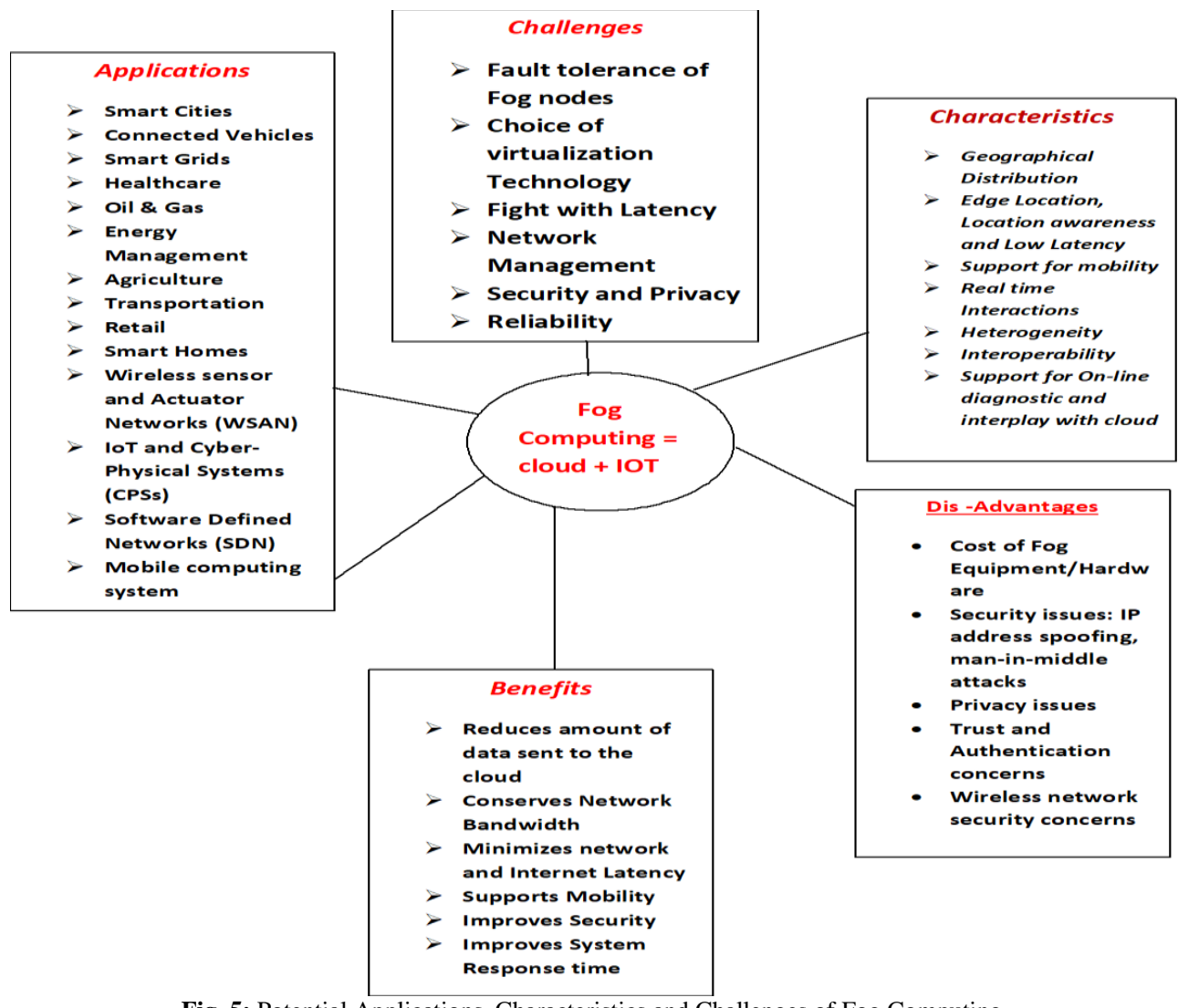

Fig. 5: Potential Applications, Characteristics and Challenges of Fog Computing. 


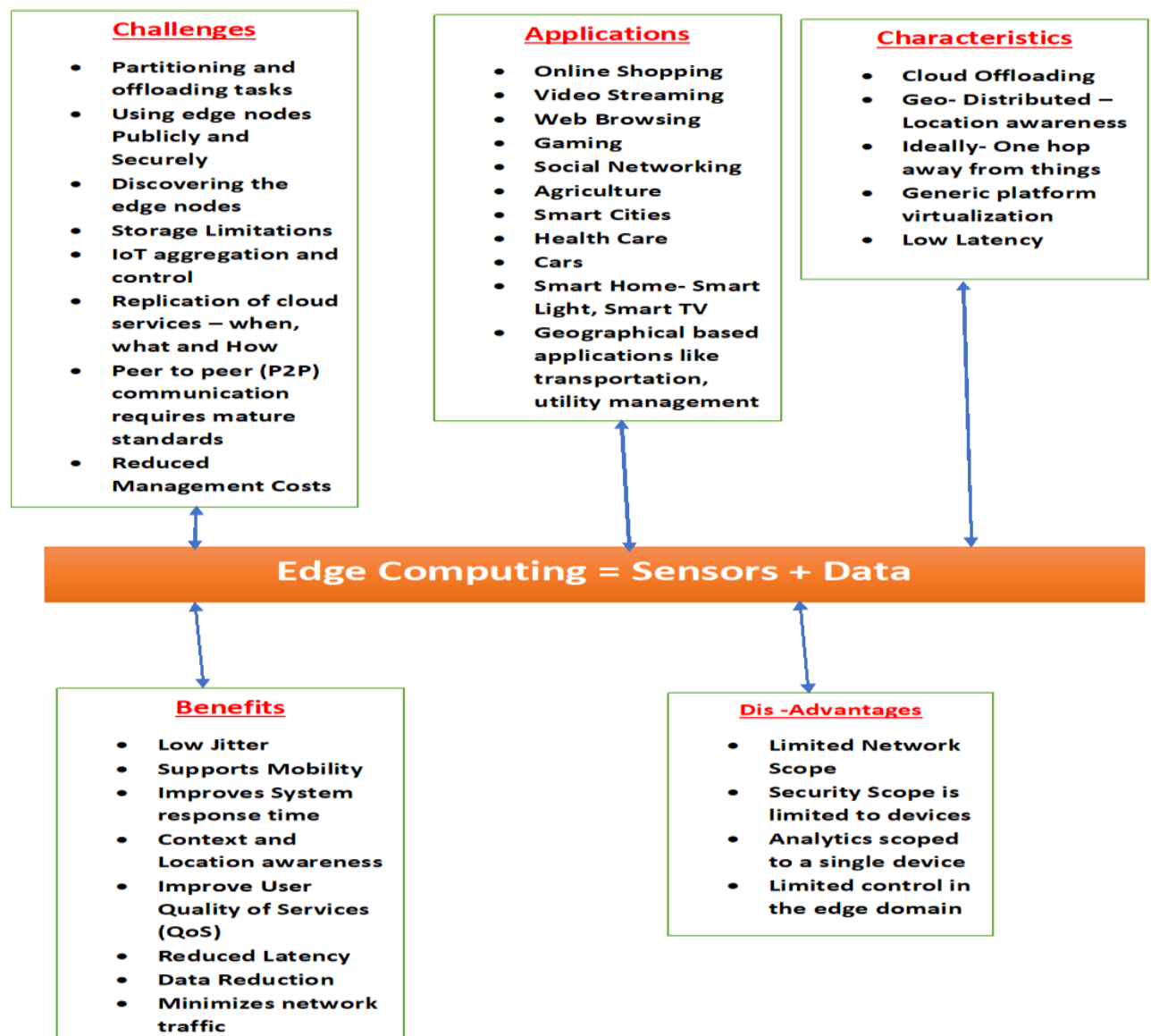

Fig. 6: Potential Applications, Characteristics and Challenges of Edge Computing.

\section{Security and privacy}

\subsection{Security}

Data security is one of the challenges in Edge and Fog Computing. Because the data generated from various devices are distributed, providing authentication and authorizing the system for the fog nodes is not an easy task. Data threats are the vulnerability in the Fog/Edge computing which affects the data and causes insecurity. Some Data Issues [15] are: Data breaches, loss of data this can be handle by using Decoy technique [16 - 18]. Two Important factors must be considered to provide security to the data:

1) Authentication: To provide security there is a need to authenticate various nodes (or) devices in the computing model. To prevent spoofing and tampering among devices, different solutions like Diffe-Hellman key exchange and Multicast authentication techniques are used.

2) Encryption: One of the effective way to achieve data security is through encryption. The Edge/Fog computing uses various encryption algorithms like RSA, Blowfish, TwoFish, Advanced Encryption standard (AES) etc. are utilized. Encryption make the original data becomes unreadable by anyone other than those who have the keys to decode it.

\subsection{Privacy}

It deals with hiding the information about devices which are used along with the time, allowing correct summary information. In Fog/ Edge Computing, preserve the location of Fog nodes and edge devices is major concern, to provide one can utilize following methods [19]:

1) Access control

In Edge and Fog computing, the access control mechanism acts as a tool to ensure the security of the system and preserving the privacy of user devices, this can be done through several key based solutions like Attribute Based Encryption mechanism [20], Policy based resource access control [21] in fog and Edge computing, to support secure collaboration and interoperability between heterogeneous resources.

2) Intrusion detection

Intrusion detection techniques are widely deployed in cloud system to mitigate attacks such as insider attack, flooding attack, port scanning, attacks on VM and hypervisor. Intrusion Detection System (IDS) can be deployed on fog node and Edge devices at network side to detect malicious attacks such as Denial-of Service (Dos), Port Scanning etc.

Other Factors may also influence Cloud, Fog and Edge Computing with Emerging Technologies, those parameters are: Resource allocation, Bandwidth allocation, latency, uninterrupted, load Balancing and new IoT security challenges. Many Algorithms are used to enhance the data in an effective way such as providing security to the data, recover loss of data, scheduling the user tasks and load balancing. For example in order to recover the data, a smart remote data backup algorithm called Seed Block Algorithm(SBA) is used, For data integrity and authentication process a digital signature called XML Signature is used to protect the sensitive data that are being sent over the cloud, For scheduling the user tasks various scheduling algorithms are used like: Min-Min, Max-Min, Enhanced Max-Min, Improved Max-Min, Cloudsim is a framework or it acts as a toolkit for modeling and simulation of cloud environment for testing and evaluating the cloud-computing model in a scalable environment, To enable migration of virtual machines across heterogeneous edges, the cloud computing model uses Flocking Behavioral Model to measure the latency, To provide security to resource nodes in a cloud Environment and predicts the trustworthiness of cloud a new manuscript called Predator prey model is used, To ensure data storage security in cloud a cryptographic protocol called on bit return protocol is used and In order to manage cloud data and utilize the resources efficiently a new container technology called Docker or Merkle model is incorporated to Fog and Edge Computing. 


\section{Conclusion}

Researchers are saying that $90 \%$ of the world's data were generated from sensors during the period of last two years i.e. the amount of data generated by IoT devices is simply huge. The current cloud model is not sufficient to handle requirements of IoT. A computing paradigm called Fog is used to perform real time analytics at the fog nodes without sending huge amount of data to cloud. To make the data processing very close to end user (or) things, a new computing model is come into existence is edge computing. The primary goal of edge computing is to perform analytics at edge of the network which reduces the network traffic, minimizes the latency and give faster response time in handling IoT data. Finally, we conclude that the Edge and Fog computing are the complements of cloud, they cannot replace it. However, Edge and fog are perfect partners for cloud and IoT while handling huge amount of data generated by sensors. The Edge Computing Paradigm benefits extends from an individual person to huge firms and the rapid development of Edge in future depends on contribution of Business Entrepreneurs, Internet Service providers, academicians, researchers and so on.

\section{References}

[1] Shubhashis Sengupta, Vikrant Kaulgud, VibhuSayanya Sharma; Cloud Computing Security- Trends and Research Directions; IEEE World Congress on Services 2011. David C. Chou, Amy Y Chou; Software as a Service (SaaS) as an outsourcing model: An economic Analysis.

[2] "Cloud Computing: A Survey on its limitations and Potential Solutions" by Mohammad Manzurul Islam , Sarwar Morshed and ParijatGoswami, IJCSI, Vol 10, issue 4, No 2, July 2013, ISSN : 1694 0784.

[3] Nisha Peter, "FOG Computing and Its Real Time Applications", IJE TAE, Volume 5, Issue 6, June 2015, ISSN 2250 - 2459.

[4] Prateeksha Varshney and Yogesh Simmhan "Demystifying Fog Computing: Characterizing Architectures, Applications and Abstractions" 21-Feb-2017.

[5] OpenFog Consortium. OpenFog Reference Architecture https://www. openfogconsortium.org/ra/, 2017. [Online; accessed 05-Sep-2017].

[6] A Survey on the Edge Computing for the Internet of Things Wei Yu, Fan Liang, Xiaofei He, William G. Hatcher, Chao Lu, Jie Lin, and Xinyu Yang. DOI 10.1109/ACCESS.2017.2778504, IEEE Access.

[7] Nebbiolo Technologies -pioneers of fog Computing -difference between Fog and Edge Computing.

[8] "Research on Key Technologies of Cloud Computing" Shufen Zhang, Hongcan Yan, Xuebin Chen, published by Elesiver, ICMPBE, 2012.

[9] "Fog Computing: Platform and Applications" Shanhe Yi, Zijiang Hao, Zhengrui Qin, and Qun Li published in IEEE Computer Society, DOI: 10.1109/HotWeb.2015.22, 2015.

[10] "Key Technologies and Application of Edge Computing" by TU Yaofeng, DONG Zhenjiang, and YANG Hongzhang, DOI 10.3969/j. issn. 1673- 5188, April 19, 2017.

[11] "A Survey on Mobile Edge Networks: Convergence of Computing, Caching and Communications" by Shuo Wang, Xing Zhang, Yan Zhang, Lin Wang, Juwo Yang, Wenbo Wang, IEEE, mar,2017.

[12] "Fog Computing: Focusing on Mobile Users at the Edge" Tom H. Luan, Longxiang Gao, Zhi Li , Yang Xiang, Guiyi We , and Limin Sun , march-2016.

[13] Shabnam Kumari, Surender Singh and Radha, "Fog Computing: Characteristics and Challenges", IJETTCS, Volume 6, Issue 2 , March- April, 2017, ISSN 2278 - 6856.

[14] Fog Computing for Sustainable Smart Cities: A Survey" by Charith Perera, Yongrui Qin, Julio C. Estrella, Stephan Reiff-Marganiec, Athanasios V. Vasilakos, ACM Computing Surveys, DOI: $0000001.0000001,2017$.

[15] "A Study on Cloud and Fog Computing Security Issues and Solutions" by Archana Lisbon A, Kavitha R, IJIRAE, Issue 3, Volume 4, ISSN: 2349-2163, March 2017.

[16] SonaliKhairnar. "Fog Computing: A New Concept To Minimize The Attacks And To Provide Security In Cloud Computing Environment," International Journal of Research in Engineering and Technology, vol. 03, no. 09, pp. 124-127, Sep. 2014.

[17] Ashwini, Thogaricheti, and Mrs Anuradha SG. "Fog Computing to protect real and sensitivity information in Cloud."
[18] Raut, Rajashri, et al. "Fog Computing Using Advance security in Cloud." International Journal of Engineering Research and Technology. Vol. 3. No. 2 (February-2014). ESRSA Publications, 2014

[19] Gao, Z., Zhu, H., Liu, Y., Li, M., Cao, Z.: Location privacy in database-driven cognitive radio networks: Attacks and countermeasures. In: INFOCOM. IEEE (2013)

[20] Yu, S., Wang, C., Ren, K., Lou, W.: Achieving secure, scalable, and fine-grained data access control in cloud computing. In: INFOCOM. IEEE (2010).

[21] Dsouza, C., Ahn, G.J., Taguinod, M.: Policy-driven security management for fog computing: Preliminary framework and a case study. In: IRI. IEEE (2014) 